\title{
Association of Japan Coma Scale score on hospital arrival with in-hospital mortality among trauma patients
}

\author{
Tetsuya Yumoto $^{1 *} \mathbb{D}$, Hiromichi Naito ${ }^{1}$, Takashi Yorifuji ${ }^{2}$, Toshiyuki Aokage ${ }^{3}$, Noritomo Fujisaki ${ }^{1}$ and Atsunori Nakao
}

\begin{abstract}
Background: The Japan Coma Scale (JCS) score has been widely used to assess patients' consciousness level in Japan. JCS scores are divided into four main categories: alert (0) and one-, two-, and three-digit codes based on an eye response test, each of which has three subcategories. The purpose of this study was to investigate the utility of the JCS score on hospital arrival in predicting outcomes among adult trauma patients.

Methods: Using the Japan Trauma Data Bank, we conducted a nationwide registry-based retrospective cohort study. Patients 16 years old or older directly transported from the trauma scene between January 2004 and December 2017 were included. Our primary outcome was in-hospital mortality. We examined outcome prediction accuracy based on area under the receiver operating characteristic curve (AUROC) and multiple logistic regression analysis with multiple imputation.

Results: A total of 222,540 subjects were included; their in-hospital mortality rate was $7.1 \%(n=15,860)$. The 10point scale JCS and the total sum of Glasgow Coma Scale (GCS) scores demonstrated similar performance, in which the AUROC (95\% Cls) showed $0.874(0.871-0.878)$ and 0.878 (0.874-0.881), respectively. Multiple logistic regression analysis revealed that the higher the JCS score, the higher the predictability of in-hospital death. When we focused on the simple four-point scale JCS score, the adjusted odds ratio (95\% confidence intervals [Cls]) were 2.31 (2.122.45), 4.81 (4.42-5.24), and 27.88 (25.74-30.20) in the groups with one-digit, two-digit, and three-digit scores, respectively, with JCS of 0 as a reference category.
\end{abstract}

Conclusions: JCS score on hospital arrival after trauma would be useful for predicting in-hospital mortality, similar to the GCS score.

Keywords: Glasgow coma scale, Japan Coma Scale, Mortality, Trauma, Traumatic brain injury

\section{Background}

Prompt and accurate assessment of severely injured trauma patients is crucial to guide proper treatment in initial management. To date, the Glasgow Coma Scale (GCS) has been widely utilized to evaluate consciousness level in a variety of clinical settings, including trauma care [1-4]. Triage protocols or outcome prediction models for trauma patients have been developed using the GCS score alone or the GCS score in combination with other clinical variables [5-7]. However, implementing the GCS presents

\footnotetext{
* Correspondence: tyumoto@cc.okayama-u.ac.jp

${ }^{1}$ Department of Emergency, Critical Care, and Disaster Medicine, Okayama University Graduate School of Medicine, Dentistry and Pharmaceutical

Sciences, 2-5-1, Shikata-cho, Kita-ku, Okayama 700-8558, Japan

Full list of author information is available at the end of the article
}

several disadvantages in terms of complexity, essential meaningfulness of the total score, and high inter-rater variability $[5,8,9]$.

In Japan, the Japan Coma Scale (JCS) is the most extensively adopted scale for assessing patients' consciousness level because of its simplicity and applicability $[10,11]$. The Japanese Diagnosis Procedure Combination database, which contains nationwide administrative claim and discharge data, includes JCS codes [12-14]. Briefly, the JCS score consists of four main categories: alert (0) and one-, two-, or three-digit codes based on degree of arousal, each of which has three subcategories. Although previous studies have indicated that JCS score correlates with outcomes

(c) The Author(s). 2019 Open Access This article is distributed under the terms of the Creative Commons Attribution 4.0 International License (http://creativecommons.org/licenses/by/4.0/), which permits unrestricted use, distribution, and 
in stroke patients $[10,15]$, the JCS has not been examined among trauma patients.

We hypothesized that consciousness level assessed using the JCS score would predict patient outcomes or identify severe traumatic brain injury (TBI) similar to or better than the GCS. A simple assessment tool using the JCS would be useful and pragmatic in the emergency department setting. The aim of this study was to examine the utility of the JCS recorded on hospital arrival in predicting in-hospital mortality in patients after trauma using a large national database, which will help raise worldwide awareness of the JCS score's applicability.

\section{Methods}

The Okayama University Hospital ethical committee approved the study (ID 1903-021). The requirement for informed consent was waived because patient data was extracted anonymously.

\section{Study design, setting, and data collection}

The design of the present study was a nationwide retrospective cohort study using registry database. We used data from a national trauma registry called the Japan Trauma Data Bank (JTDB), which has been described in detail elsewhere $[7,11]$. Briefly, the JTDB was established in 2003 with the Committee for Clinical Care Evaluation of the Japanese Association for Acute Medicine and the Trauma Surgery Committee of the Japanese Association for the Surgery of Trauma. As of March 2018, 272 secondary and tertiary emergency and critical care centers participated in Japanese trauma care and research, from which data patients with Abbreviated Injury Scale (AIS) scores of 3 or above were continuously recorded [16]. The database contains patient demographics, mechanism of injury, vital signs, and consciousness scale ratings based on JCS and the GCS scores on arrival, AIS scores, Injury Severity Score (ISS), treatment, and survival status at hospital discharge. We collected the data from the database, which included data of all trauma patients between 2004 and 2017, provided by the Japan Trauma Care and Research.

\section{Participants}

All patients with trauma who were 16 years old or older directly transported from the injury scene between January 2004 and December 2017 were included in this study. Patients with burn injuries, in cardiac arrest at the scene or on arrival, those with AIS scores of 6 in any region, or those undergoing interfacility transport were excluded.

\section{Japan Coma Scale}

The JCS was first reported in 1974 [17]. The scale is composed of four main categories: 0 and one-, two-, and three-digit codes corresponding with alert, awake without stimuli, arousable with some stimuli (but reverts to previous status if stimulus stops), and unarousable by any forceful stimuli, respectively. Each code is further divided into three subcategories: 1,2 and 3 in the one-digit code, 10, 20, and 30 in the two-digit code, and 100, 200, and 300 in the three-digit code. Hence, there are 10 grades in total (Additional file 1: Table S1). A JCS score of 0 is equal to a GCS score of 15 (E4V5M6), while a JCS score of 300 corresponds with a GCS score of 3 (E1V1M1).

\section{Outcome measures}

The primary outcome was in-hospital mortality from all causes. The secondary outcome was the presence of severe TBI, which was defined as head AIS scores of 4 or 5 .

\section{Statistical analysis}

Continuous variables are presented as median and interquartile range values, whereas categorical variables are shown as frequencies and percentages. To account for missing data, multiple imputation was performed to analyze incomplete data, replacing each missing value with a set of 20 substitute plausible values $[18,19]$. Covariables, including sex, mechanism of injury, systolic blood pressure, heart rate, respiratory rate, JCS score, GCS score, ISS, and hospital mortality were used to develop 20 complete data sets. Using the pooled data, we examined ability to accurately predict outcomes based on area under the receiver operating characteristic curve (AUROC) and multiple logistic regression analysis, focusing on JCS and GCS scores. As mentioned above, the JCS score has four main categories with three subcategories except for the JCS score of 0 ; the simple four JCS categories as well as the 10-point JCS scale were evaluated simultaneously [10]. GCS scores were evaluated using subscores, including those for eye, verbal, and motor responses, in addition to a simple sum score. Adjusted odds ratios (ORs) and their 95\% confidence intervals (CIs) for primary outcomes were obtained after adjusting for age (16-39 vs. $40-64$ vs. $\geq 65)$; gender; mechanism of injury (blunt or other); systolic blood pressure of < $90 \mathrm{mmHg}$ vs. $\geq 90 \mathrm{mmHg}$; heart rate of < $120 \mathrm{bpm}$ vs. $\geq 120 \mathrm{bpm}$; respiratory rate of $\leq 9 \mathrm{cpm}$ vs. $10-29 \mathrm{cpm}$ vs. $\geq 30 \mathrm{cpm}$; presence or absence of severe TBI (head AIS score of 4 or 5); presence or absence of emergency surgical intervention (craniotomy, thoracotomy, laparotomy, or angioembolization); and ISS score of $\leq 8$ vs. $9-15$ vs. $\geq 16$. Adjusted ORs and their $95 \%$ CIs for secondary outcome were obtained using the same variables as the primary outcome except for the presence or absence of severe TBI and emergency surgical intervention and ISS. The suggested cut-off value for each score was determined using the Youden index. Complete 
cases were also assessed using a sensitivity analysis for in-hospital mortality. A two-tailed $P$ value of $<0.05$ was considered statistically significant. All analyses were conducted using IBM SPSS Statistics 25 (IBM SPSS, Chicago, IL, U.S.A.).

\section{Results}

A total of 222,540 trauma patients meeting the inclusion criteria were identified and included in this study (Fig. 1). Study population characteristics are summarized in Table 1. Median age and ISS were 62 and 10, respectively. Of the 222,540 patients, 49,609 (22.3\%), 21,388 (9.6\%), and 19,424 (8.7\%) had missing JCS scores, GCS scores, and in-hospital mortality data, respectively. Complete case demographics are shown in the supplemental material (Additional file 2: Table S2). Regarding consciousness level, 118,158 (53.1\%) patients exhibited altered mental status (JCS $\geq 1$ ) according to JCS score. Overall in-hospital mortality was $7.1 \%(n=15,860)$. Distribution of in-hospital mortality percentages based on JCS and GCS scores are shown in Fig. 2. The higher the JCS score or the lower the GCS score, the higher the inhospital mortality rate observed.

Data presented in Table 2 shows the performances of both the JCS score and GCS score in predicting in-hospital mortality. AUROC of greater than 0.8 was achieved in both scores. The 10-point scale JCS and the total sum of GCS scores demonstrated similar performance, of which the AUROC (95\% CIs) was $0.874(0.871-0.878)$ and 0.878 (0.874-0.881), and its suggested cut-off value was 3 on the JCS score and 11 on the GCS score, respectively.

Table 3 shows the results of multiple logistic regression analysis for in-hospital mortality focusing on JCS and GCS scores. The higher the JCS 10-point scale score, the higher the predictability of in-hospital death. When we focused on the simple four-point scale JCS score ( 0 , one-digit, two-digit, and three-digit scores), the adjusted ORs (95\% CIs) were 2.31 (2.12-2.45), 4.81 (4.42-5.24), and 27.88 (25.74-30.20) in the groups with one-digit, two-digit, and three-digit scores, respectively, with JCS score of 0 as a reference category. As for GCS score based on the individual component and the total sum of three subscores, lower scores were significantly associated with higher mortality. The eye component showed the highest ability to predict in-hospital death, of which adjusted ORs (95\% CIs) were 2.55 (2.54-2.56) for every one unit decrease in the score.

As for secondary outcome measures, the 10-point scale JCS score demonstrated the highest predictability for severe TBI, in which the AUROC (95\% CIs) was 0.781 (0.7780.783 ) with a suggested cut-off value of 2 (Additional file 3 : Table S3). In multiple logistic regression analysis, there was a trend toward significance of the association of higher JCS scores with higher predictability of severe TBI (Additional file 4: Table S4). Regarding the GCS score, the eye component showed the highest ability to predict severe TBI, of which adjusted ORs (95\% CI) were 2.69 (2.68-2.69) for every one unit decrease in the score.

In sensitivity analysis for in-hospital mortality with complete cases, results were similar to those from the multiple imputation analysis (Additional files 5 and 6: Tables S5 and S6).

\section{Discussion}

In this large Japanese cohort study, we found that assessment of consciousness level based on JCS score at

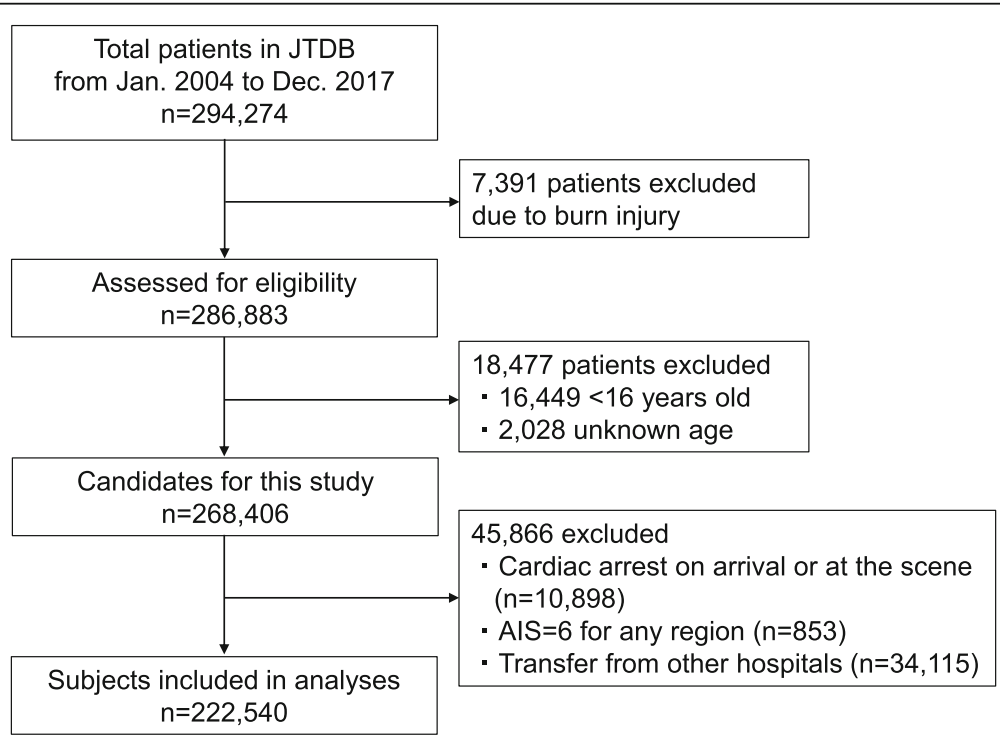

Fig. 1 Flow diagram of the study populationJTDB: Japan Trauma Data Bank; AIS: Abbreviated Injury Scale. 
Table 1 Study population characteristics

\begin{tabular}{|c|c|}
\hline & Overall $(n=222,540)$ \\
\hline Age (years), median (IQR) & $62(41,77)$ \\
\hline 16-39 (years), n (\%) & $53,071(23.8)$ \\
\hline 40-64 (years), n (\%) & $65,572(29.5)$ \\
\hline$\geq 65$ (years), n (\%) & $103,897(46.7)$ \\
\hline Male, n (\%) & $138,023(62.0)$ \\
\hline Blunt mechanism injury, n (\%) & $213,280(95.8)$ \\
\hline Systolic blood pressure (mmHg), median (IQR) & $137(117,158)$ \\
\hline$<90 \mathrm{mmHg}, \mathrm{n}(\%)$ & $15,672(7.0)$ \\
\hline Heart rate (bpm), median (IQR) & $83(72,96)$ \\
\hline$\geq 120$ bpm, n (\%) & $12,883(5.8)$ \\
\hline Respiratory rate (cpm), median (IQR) & $20(17,24)$ \\
\hline $10-29$ (cpm), n (\%) & $196,595(88.4)$ \\
\hline$<10(\mathrm{cpm}), \mathrm{n}(\%)$ & $3380(1.5)$ \\
\hline$\geq 30$ (cpm), n (\%) & $22,565(10.1)$ \\
\hline \multicolumn{2}{|l|}{ Japan Coma Scale } \\
\hline $0, \mathrm{n}(\%)$ & $104,382(46.9)$ \\
\hline $1, \mathrm{n}(\%)$ & $37,934(17.0)$ \\
\hline $2, \mathrm{n}(\%)$ & $21,697(9.7)$ \\
\hline $3,(\%)$ & $13,929(6.3)$ \\
\hline $10, \mathrm{n}(\%)$ & $14,875(6.7)$ \\
\hline $20, n(\%)$ & $4320(1.9)$ \\
\hline $30, n(\%)$ & $3549(1.6)$ \\
\hline $100, n(\%)$ & $5484(2.5)$ \\
\hline $200, n(\%)$ & $6810(3.1)$ \\
\hline $300, \mathrm{n}(\%)$ & $9560(4.3)$ \\
\hline One-digit $(1,2,3), n(\%)$ & $73,560(33.1)$ \\
\hline Two-digit $(10,20,30), n(\%)$ & $22,744(10.2)$ \\
\hline Three-digit $(100,200,300), n(\%)$ & $21,854(9.8)$ \\
\hline Glasgow Coma Scale, median (IQR) & $15(13,15)$ \\
\hline$\leq 8, \mathrm{n}(\%)$ & $22,514(10.1)$ \\
\hline Eye response, median (IQR) & $4(3,4)$ \\
\hline Verbal response, median (IQR) & $5(4,5)$ \\
\hline Motor response, median (IQR) & $6(6,6)$ \\
\hline Craniotomy, n (\%) & $7165(3.2)$ \\
\hline Head AIS score of 4 or $5, n(\%)$ & $39,151(17.6)$ \\
\hline Isolated severe TBI, n (\%) & $9849(4.4)$ \\
\hline \multicolumn{2}{|l|}{ Surgical or hemostatic intervention, n (\%) } \\
\hline Thoracotomy, n (\%) & $2033(0.9)$ \\
\hline Laparotomy, n (\%) & $5976(2.7)$ \\
\hline Angioembolization, n (\%) & $5879(2.6)$ \\
\hline Injury Severity Score, median (IQR) & $10(9,18)$ \\
\hline$\leq 8, \mathrm{n}(\%)$ & $42,332(19.0)$ \\
\hline $9-15, \mathrm{n}(\%)$ & $92,531(41.6)$ \\
\hline $16 \leq, \mathrm{n}(\%)$ & $87,677(39.4)$ \\
\hline
\end{tabular}

Table 1 Study population characteristics (Continued)

\begin{tabular}{ll}
\hline In-hospital mortality, $\mathrm{n}(\%)$ & Overall $(n=222,540)$ \\
\hline IQR Interquartile range, AIS Abbreviated Injury Scale, TBI traumatic brain injury
\end{tabular}

hospital arrival would be useful for predicting inhospital mortality and severe TBI among adult trauma patients. The JCS score may be similar or even superior to the GCS score in accuracy and simplicity for evaluation of consciousness level.

For decades, the JCS has been used in various emergency settings to assess patients' consciousness levels in Japan [10, 11, 20-23]. Shigematsu et al. emphasized several advantages of the JCS, including its simplicity, reliability, and applicability [10]. The JCS has four main categories that can be subdivided using the eye response test alone. The GCS is a three-axis scale evaluating eye, verbal, and motor responses, while the JCS is a one-axis scale. Therefore, the same total GCS scores are associated with quite different outcomes, indicating that the total GCS score is meaningless [24]. Our current data showed a correlation between the four-point scale JCS score predictability of in-hospital death and severe TBI. As a two-digit JCS score encompasses E2 and E3 in GCS, the four-point JCS scale would be much simpler, similar to the GCS. Although the 10-point scale JCS assessed verbal or motor responses, it enabled a more detailed evaluation of predictability of in-hospital mortality, as shown in Table 2.

Given the verbal score limitations in intubated patients and complexity of calculating total GCS score, the Glasgow motor scale alone has been investigated for outcome prediction or as a triage test, in which the simplified motor scale has been revealed to have higher predictive power as well as higher interrater reliability $[5,8,25]$. Notably, our results of AUROC $>0.8$ demonstrated that either the simplified four-point scale or 10point scale JCS scores had strong predictive accuracy for in-hospital death, similar to the Glasgow motor scale.

Nonetheless, only a few studies have examined the utility of the JCS score. A previous study showed that simple four-point scale JCS score at onset of stroke significantly correlated with Activities of Daily Living levels at 30 days after the event [10]. Another study described the usefulness of the JCS score in TBI patients in deciding to perform head computed tomography [22]. However, the latter study involved a relatively small sample after mild head injury without evaluating the association between JCS score and clinical outcomes.

To our knowledge, this is the first study to examine the ability of the JCS score to predict mortality and severe TBI among trauma patients. The strength of our study lies in its large cohort from multi-center 

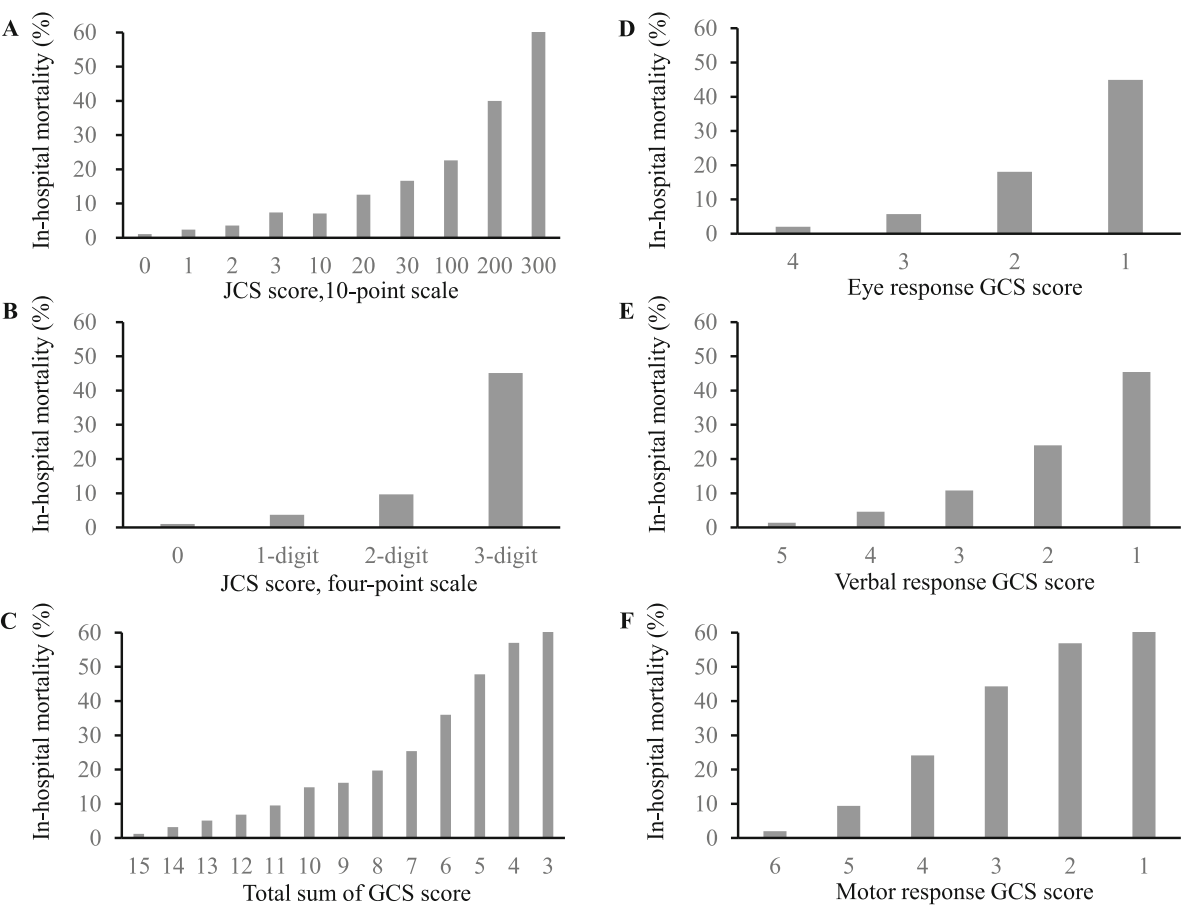

Fig. 2 Percentage distribution of in-hospital mortality based on Japan Coma Scale (a, b) and Glasgow Coma Scale scores (c-f)

institutions nationwide, in which we showed the outcome predictability of the 10-point scale JCS as well as the simple four-point JCS. Quick and simple assessment of consciousness level using the JCS will help clinicians identify "red flag" patients immediately.

Our study has several limitations. First, a substantial amount of patient data was missing, including JCS score and mortality. To account for this limitation, multiple imputation was used, in which we confirmed that the same results were obtained as the complete cases. Second, the interrater variation of the JCS score was not evaluated. Although the simple four-point scale JCS could provide consistency among examiners, the 10point scale might have had variations to some extent as the GCS score had $[9,10]$. Nevertheless, the JCS and the GCS assessments were found to be well correlated $[22,26]$. Finally, this study was conducted in Japan, where the assessment using JCS has been widespread. An international study should be designed to validate our results.

\section{Conclusions}

In summary, assessment of consciousness level using JCS score on hospital arrival was an efficient predictor of in-hospital mortality among adult trauma patients, similar to the GCS score. This result suggested that the JCS score could be useful and applicable as a triage tool in trauma care settings. Further international studies are warranted to confirm our results.

Table 2 Predictive performance of the Japan Coma Scale and the Glasgow Coma Scale for in-hospital mortality

\begin{tabular}{|c|c|c|c|c|c|c|}
\hline & AUROC $(95 \% \mathrm{Cl})$ & Cut-off & Sensitivity, \% & Specificity, \% & PPV, \% & NPV, \% \\
\hline JCS score, 10-point scale & $0.874(0.871-0.878)$ & 3 & 82.5 & 78.0 & 22.4 & 98.3 \\
\hline JCS score, four-point scale & $0.859(0.856-0.863)$ & 2-digit & 76.0 & 84.3 & 27.0 & 97.9 \\
\hline Eye response GCS score & $0.829(0.825-0.833)$ & 2 & 65.7 & 92.2 & 39.3 & 97.2 \\
\hline Verbal response GCS score & $0.860(0.857-0.864)$ & 3 & 75.0 & 87.9 & 32.2 & 97.9 \\
\hline Motor response GCS score & $0.842(0.838-0.846)$ & 5 & 77.5 & 84.0 & 27.1 & 98.0 \\
\hline Total sum of GCS score & $0.878(0.874-0.881)$ & 11 & 74.7 & 88.5 & 33.3 & 97.9 \\
\hline
\end{tabular}

AUROC Area under the receiver operating characteristic curve, PPV Positive predictive value, NPV Negative predictive value, JCS Japan Coma Scale, GCS Glasgow Coma Scale 
Table 3 Multiple logistic regression analysis for in-hospital mortality focusing on the Japan Coma Scale (10-point and fourpoint scale) and the Glasgow Coma Scale (eye, verbal, and motor response scores and total sum scores) on arrival

\begin{tabular}{llll}
\hline & Adjusted ORs & $95 \% \mathrm{Cls}$ & $P$-valu \\
\cline { 2 - 2 } & & \\
0 & Reference & & \\
1 & 1.03 & $0.69-1.53$ & 0.899 \\
2 & 1.65 & $1.32-2.01$ & $<0.001$ \\
3 & 2.19 & $1.78-2.70$ & $<0.001$ \\
10 & 3.90 & $3.15-4.83$ & $<0.001$ \\
20 & 3.70 & $2.99-4.57$ & $<0.001$ \\
30 & 6.26 & $5.03-7.79$ & $<0.001$ \\
100 & 8.00 & $6.26-10.21$ & $<0.001$ \\
200 & 11.36 & $9.22-14.00$ & $<0.001$ \\
300 & 23.95 & $19.30-29.71$ & $<0.001$
\end{tabular}

JCS score, four-point scale

one-digit $\quad 2.31$

two-digit $\quad 4.81$

three-digit $\quad 27.88$

$2.12-2.52$

$<0.001$

$4.42-5.24$

$<0.001$

$25.74-30.20$

$<0.001$

Eye response GCS score

E $\quad 2.55$

$2.54-2.56$

Verbal response GCS score

$\mathrm{V}$

$2.18-2.19$

Motor response GCS score

M 2.01

2.0

Total sum of GCS score

$\mathrm{E}+\mathrm{V}+\mathrm{M}$

1.38

$1.38-1.38$

ORs Odds ratios, Cls Confidence intervals, JCS Japan Coma Scale, GCS Glasgow Coma Scale, TBI Traumatic brain injury, ISS Injury Severity Score

Each adjusted ORs and their $95 \% \mathrm{Cls}$ were obtained after adjusting for age (16-39 vs. $40-64$ vs. $\geq 65$ ); gender; mechanism of injury (blunt or others); systolic blood pressure of $<90 \mathrm{mmHg}$ vs. $\geq 90 \mathrm{mmHg}$; heart rate of $<120 \mathrm{bpm}$ vs. $\geq 120 \mathrm{bpm}$; respiratory rate of $\leq 9 \mathrm{cpm}$ vs. $10-29 \mathrm{cpm}$ vs. $\geq 30$ $\mathrm{cpm}$; presence or absence of severe TBI (head AIS scores of 4 or 5); presence or absence of emergency surgical intervention (craniotomy, thoracotomy, laparotomy, or angioembolization); and ISS of $\leq 8$ vs. $9-15$ vs. $\geq 16$. The adjusted ORs of the GCS (eye, verbal, and motor response scores and total sum scores) represent the increase in odds of the outcome with every one unit decrease in the score

\section{Supplementary information}

Supplementary information accompanies this paper at https://doi.org/10. 1186/s12873-019-0282-x.

Additional file 1: Table S1. Japan Coma Scale scoring.

Additional file 2: Table S2. Demographics of complete cases.

Additional file 3: Table S3. Predictive performance of the Japan Coma Scale and the Glasgow Coma Scale for severe TBI.

Additional file 4: Table S4. Multiple logistic regression analysis for severe traumatic brain injury focusing on Japan Coma Scale score and Glasgow Coma Scale score on arrival.
Additional file 5: Table S5. Predictive performance of the Japan Coma Scale and the Glasgow Coma Scale for in-hospital mortality among complete cases.

Additional file 6: Table S6. Multiple logistic regression analysis for inhospital mortality focusing on the Japan Coma Scale (10-point and fourpoint scale) and the Glasgow Coma Scale (eye, verbal, and motor responses and total sum) on arrival among complete cases.

\section{Abbreviations}

AIS: Abbreviated Injury Scale; AUROC: Area under the receiver operating characteristic curve; Cls: Confidence intervals; GCS: Glasgow Coma Scale; ISS: Injury Severity Score; JCS: Japan Coma Scale; JTDB: Japan Trauma Data Bank; ORs: Odds ratios; TBI: Traumatic brain injury

\section{Acknowledgements}

We express our gratitude to all the JTDB registry participants and members of the Trauma Registry Committee (Japanese Association for Trauma Surgery) and the Committee for Clinical Care Evaluation (Japanese Association for Acute Medicine).

\section{Authors' contributions}

TYu, HN, and TYo conceived and designed the study. TYu, HN, TA, and NF conducted data collection. TYu, HN, and TYo analyzed and interpreted the data. TYu, TYo, and AN drafted the manuscript. AN takes responsibility for the paper as a whole. All authors have read and approved the final version of the manuscript.

\section{Funding}

No funding sources supported the study described in this manuscript.

\section{Availability of data and materials}

The data that support the findings of this study are available from JTDB, but restrictions apply to the availability of these data, which were used under license for the current study, and so are not publicly available. Data are however available from the authors upon reasonable request and with permission of JTDB

\section{Ethics approval and consent to participate}

The study was conducted according to the Helsinki Declaration and approved by the Okayama University Hospital ethical committee (ID 1903-021).

\section{Consent for publication}

Not applicable.

Competing interests

The authors declare that they have no competing interests.

\section{Author details}

'Department of Emergency, Critical Care, and Disaster Medicine, Okayama University Graduate School of Medicine, Dentistry and Pharmaceutical Sciences, 2-5-1, Shikata-cho, Kita-ku, Okayama 700-8558, Japan. ${ }^{2}$ Department of Epidemiology, Okayama University Graduate School of Medicine, Dentistry and Pharmaceutical Sciences, Okayama, Japan. ${ }^{3}$ Department of Geriatric Emergency Medicine, Okayama University Graduate School of Medicine, Dentistry and Pharmaceutical Sciences, Okayama, Japan.

Received: 26 June 2019 Accepted: 24 October 2019

Published online: 06 November 2019

\section{References}

1. Teasdale G, Maas A, Lecky F, Manley G, Stocchetti N, Murray G. The Glasgow coma scale at 40 years: standing the test of time. Lancet Neurol. 2014;13: 844-54.

2. Davis DP, Serrano JA, Vilke GM, Sise MJ, Kennedy F, Eastman AB, et al. The predictive value of field versus arrival Glasgow coma scale score and TRISS calculations in moderate-to-severe traumatic brain injury. J Trauma. 2006:60: 985-90. 
3. Singh B, Murad MH, Prokop LJ, Erwin PJ, Wang Z, Mommer SK, et al. Metaanalysis of Glasgow coma scale and simplified motor score in predicting traumatic brain injury outcomes. Brain Inj. 2013;27:293-300.

4. Davis JW, Dirks RC, Sue LP, Kaups KL. Attempting to validate the overtriage/ undertriage matrix at a level I trauma center. J Trauma Acute Care Surg. 2017:83:1173-8.

5. Brown JB, Forsythe RM, Stassen NA, Peitzman AB, Billiar TR, Sperry $J$, et al. Evidence-based improvement of the National Trauma Triage Protocol: the Glasgow coma scale versus Glasgow coma scale motor subscale. J Trauma Acute Care Surg. 2014;77:95-102.

6. Kong G, Yin X, Wang T, Body R, Chen YW, Wang J, et al. Current state of trauma care in China, tools to predict death and ICU admission after arrival to hospital. Injury. 2015;46:1784-9.

7. Kimura A, Tanaka N. Reverse shock index multiplied by Glasgow coma scale score ( $\mathrm{rSIG}$ ) is a simple measure with high discriminant ability for mortality risk in trauma patients: an analysis of the Japan trauma data Bank. Crit Care. 2018;22:87.

8. Healey C, Osler TM, Rogers FB, Healey MA, Glance LG, Kilgo PD, et al. Improving the Glasgow coma scale score: motor score alone is a better predictor. J Trauma. 2003;54:671-80.

9. Gill MR, Reiley DG, Green SM. Interrater reliability of Glasgow coma scale scores in the emergency department. Ann Emerg Med. 2004:43:215-23.

10. Shigematsu $\mathrm{K}$, Nakano $\mathrm{H}$, Watanabe $\mathrm{Y}$. The eye response test alone is sufficient to predict stroke outcome--reintroduction of Japan Coma Scale: a cohort study. BMJ Open. 2013;3:e002736.

11. Yumoto T, Mitsuhashi T, Yamakawa Y, lida A, Nosaka N, Tsukahara K, et al. Impact of Cushing's sign in the prehospital setting on predicting the need for immediate neurosurgical intervention in trauma patients: a nationwide retrospective observational study. Scand J Trauma Resusc Emerg Med. 2016;24:147.

12. Wada $T$, Yasunaga $H$, Inokuchi $R$, Matsui $H$, Matsubara $T$, Ueda $Y$, et al. Effectiveness of surgical rib fixation on prolonged mechanical ventilation in patients with traumatic rib fractures: a propensity score-matched analysis. J Crit Care. 2015:30:1227-31.

13. Wada T, Yasunaga H, Yamana H, Matsui H, Matsubara T, Fushimi K, et al. Development and validation of a new ICD-10-based trauma mortality prediction scoring system using a Japanese national inpatient database. Inj Prev. 2017;23:263-7.

14. Wada T, Yasunaga H, Doi K, Matsui H, Fushimi K, Kitsuta $Y$, et al. Relationship between hospital volume and outcomes in patients with traumatic brain injury: a retrospective observational study using a national inpatient database in Japan. Injury. 2017;48:1423-31.

15. Kurogi R, Kada A, Nishimura K, Kamitani S, Nishimura A, Sayama T, et al. Effect of treatment modality on in-hospital outcome in patients with subarachnoid hemorrhage: a nationwide study in Japan (J-ASPECT study). J Neurosurg. 2018;128:1318-26.

16. Japan Trauma Care and Research. Japan Trauma Data Bank Report 2018 https://www.jtcr-jatec.org/traumabank/dataroom/data/JTDB2018e.pdf. Accessed 1 June 2019

17. Ohta T, Waga S, Handa W, Saito I, Takeuchi K. New grading of level of disordered consciousness (author's transl). No shinkei geka. Neurol Surg. 1974;2:623-7.

18. Rubin DB, Schenker N. Multiple imputation in health-care databases: an overview and some applications. Stat Med. 1991;10:585-98.

19. Janssen KJ, Donders AR, Harrell FE Jr, Vergouwe Y, Chen Q, Grobbee DE, et al. Missing covariate data in medical research: to impute is better than to ignore. J Clin Epidemiol. 2010;63:721-7.

20. Suzuki S, Sano K, Handa H, Asano T, Tamura A, Yonekawa Y, et al. Clinical study of OKY-046, a thromboxane synthetase inhibitor, in prevention of cerebral vasospasms and delayed cerebral ischaemic symptoms after subarachnoid haemorrhage due to aneurysmal rupture: a randomized double-blind study. Neurol Res. 1989;11:79-88.

21. Sakai K, Iwahashi K, Terada K, Gohda Y, Sakurai M, Matsumoto Y. Outcome after external decompression for massive cerebral infarction. Neurol Med Chir (Tokyo). 1998;38:131-6.

22. Ono K, Wada K, Takahara T, Shirotani T. Indications for computed tomography in patients with mild head injury. Neurol Med Chir (Tokyo). 2007:47:291-7.

23. Irisawa T, Iwami T, Kitamura T, Nishiyama C, Sakai T, Tanigawa-Sugihara K, et al. An association between systolic blood pressure and stroke among patients with impaired consciousness in out-of-hospital emergency settings. BMC Emerg Med. 2013;13:24.
24. Teoh LS, Gowardman JR, Larsen PD, Green R, Galletly DC. Glasgow coma scale: variation in mortality among permutations of specific total scores. Intensive Care Med. 2000;26:157-61.

25. Gill M, Martens K, Lynch EL, Salih A, Green SM. Interrater reliability of 3 simplified neurologic scales applied to adults presenting to the emergency department with altered levels of consciousness. Ann Emerg Med. 2007:49:403-7.

26. Todo T, Usui M, Takakura K. Treatment of severe intraventricular hemorrhage by intraventricular infusion of urokinase. J Neurosurg. 1991;74:81-6.

\section{Publisher's Note}

Springer Nature remains neutral with regard to jurisdictional claims in published maps and institutional affiliations.
Ready to submit your research? Choose BMC and benefit from:

- fast, convenient online submission

- thorough peer review by experienced researchers in your field

- rapid publication on acceptance

- support for research data, including large and complex data types

- gold Open Access which fosters wider collaboration and increased citations

- maximum visibility for your research: over $100 \mathrm{M}$ website views per year

At BMC, research is always in progress.

Learn more biomedcentral.com/submissions 